\title{
Outcome of temporalis fascia myringoplasty with and without use of platelet rich plasma: A randomized control trial
}

\author{
pradipta parida $^{1}$, Prity Sharma ${ }^{1}$, Chappity Preetam ${ }^{1}$, Anindya Nayak ${ }^{1}$, Somnath \\ Mucherjee $^{1}$, and Pradeep Pradhan ${ }^{1}$
}

${ }^{1}$ All India Institute of Medical Sciences Bhubaneswar

May 28, 2020

\begin{abstract}
Objectives: To compare the efficacy of temporalis fascia myringoplasty using platelet rich plasma(PRP) $\{\mathrm{MP}-\mathrm{PRP}\}$ and conventional temporalis fascia myringoplasty(MP-C) in treatment of moderate to large central tympanic membrane(TM) perforation . STUDY DESIGN: Randomized-Control-Trial SETTING: Tertiary-Health-Centre SUBJECTS AND METHODS:We randomly assigned eighty patients with COM-mucosal-type with medium to large central TM perforation and conductive hearing loss planned for primary myringoplasty to receive either MP-PRP orMP-C. Myringoplasty was performed through post-auricular approach underlay graft using temporalis fascia. Primary outcome was graft uptake (an intact TM) at 6 months postoperatively. Secondary outcome was the post-operative hearing improvement measured by pure-tone-audiometry (PTA). RESULTS: Eighty myringoplasties (MP-PRP group-40, MP-C group-40) done for 80 patients ( $m a l e=41$; female=39, age-group=18-45years) were included in analysis. At 6 months postoperatively graft uptake rate was $94.4 \%$ in MP-PRP and $92.1 \% \%$ in MP-C group. There was no statistically significant difference in graft uptake between the two groups( $\mathrm{p}=0.358)$. Success in terms of hearing gain ([?]10dB) was achived in 34 patients (89.5\%) in MP-C and 37 patients (94.9\%) in MP-PRP group.At 6months follow-up; mean-PTA-average improved from $35.10 \pm 5.401 \mathrm{~dB}$ to $27.74 \pm 5.660 \mathrm{~dB}$ and mean ABG improved from $24.00 \pm 5.204 \mathrm{~dB}$ to17.42 $\pm 5.559 \mathrm{~dB}$ in MP-C group. At 6months follow-up; mean-PTA-average improved from $37.00 \pm 6.144 \mathrm{~dB}$ to $26.65 \mathrm{~dB}$ and mean air bone gap(ABG) improved from $25.98 \pm 5.736 \mathrm{~dB}$ to $16.21 \pm 4.318 \mathrm{~dB}$ in MP-PRPgroup. No statistically significant differences in improvement in PTA-values were observed between both groups. $(\mathrm{p}=0.336)$.Postoperative complications were similar in both groups. Conclusion: Graft uptake, hearing outcomes and complications of MP-PRP were similar to MP-C. MP-PRP offers no advantages over MP-C for treatment of TM perforation.
\end{abstract}

\section{Hosted file}

Manuscript_final.doc available at https://authorea.com/users/327129/articles/454805-outcomeof-temporalis-fascia-myringoplasty-with-and-without-use-of-platelet-rich-plasma-arandomized-control-trial 